Bei diesen Untersuchungen haben mich die Herren Apotheker Dr. E. Amort und A. Gronover, welche beide am hiesigen Institute als Assistenten thätig sind, in dankenswerter Weise unterstützt. Ich benutze gern die Gelegenheit, den beiden Herren auch an dieser Stelle den besten Dank für ihre mit vielen Unannehmlichkeiten verknüpfte äusserst mühselige Mitarbeit auszusprechen.

\title{
I. Ueber die Einwirkung von Arsenwasserstoff auf Quecksilberchlorid.
}

\author{
Von A. Partheil und E. Amort.
}

Die Einwirkung von Arsenwasserstoff auf Quecksilberchlorid ist sowohl in wässeriger, wie in alkoholischer Lösung schon mehrfach Gegenstand von Untersuchungen gewesen.

Stromeyer ${ }^{1}$ ) beobachtete, dass sich bei der Einwirkung der beiden Reagentien aufeinander zunächst arsenige Säure und Quecksilberchlorid bilde, dass das Endprodukt der Reaktion aber ein Amalgam aus Quecksilber und Arsenik sei. Soubeiran²) stellte ebenfalls eine Einwirkung von Arsenwasserstoff auf wässerige Quecksilberchloridlösung fest; er geht aber auf die dabei entstehenden Produkte nicht näher ein.

H. Ros $\mathrm{e}^{3}$ ) stellte durch Einleiten von Arsenwasserstoff in wässerige Quecksilberchloridlösung einen in getrocknetem Zustande braungelben Körper dar, dem er die Formel $\mathrm{As}^{2} \mathrm{Hg}^{3}+3 \mathrm{HgCl}^{2}$ zuerteilte und dessen Eigenschaften er eingehend beschrieben hat. Er scheint der Meinung zu sein, mit seiner Arbeit die Angaben von Stromeyer widerlegt zu habeu. Lohmann ${ }^{4}$ ) hat den von Rose beschriebenen Körper sowohl aus wässeriger, als auch namentlich leicht aus alkoholischer Lösung erhalten und bestätigt die Angaben Rose's hinsichtlich des analytischen Befundes. Er giebt ihm die Formel $\mathrm{AsHg}^{8} \mathrm{Cl}^{3}$.

Franceschi ${ }^{5}$ ) dagegen fand, dass beim Einleiten von Arsenwasserstoff in Quecksilberchloridlösung zunächst ein gelber Körper entsteht, für welchen er die Formel $\mathrm{AsH}\left(\mathrm{HgCl}^{2}\right.$ ermittelte.

1) G melin, Handbuch, III. Aufl. 1826, 921.

2) Poggend. Ann. 19, 191.

3) Ebenda 51, 423.

4) Pharm. Ztg. 1891, No. 95.

5) L'Orosi 13, 289. 
Unsere Untersuchungen haben zunächst im grossen und ganzen die Richtigkeit aller dieser in der Litteratur vorhandenen Angaben, anch derer von Stromeyer ergeben, sie haben aber weiter gezeigt, dass damit die Reihe der Körper noch nicht erschöpft war, welche bei der Einwirkung von Arsenwasserstoff auf Quecksilberchlorid entstehen können.

Zunächst verläuft die Reaktion derartig, dass unter gleichzeitiger Bildung von Salzsäure der Reihe nach die drei Wasserstoffatome des Arsenwasserstoffs durch $\mathrm{HgCl}$ ersetzt werden, sodass die Körper:

As $\mathrm{H}^{2} \mathrm{Hg} \mathrm{Cl}$, Einfach-Chlorquecksilberarsin, AsH (HgCl)2, Zweifach-Chlorquecksilberarsin, As (Hg Cl) , Dreifach-Chlorquecksilberarsin,

entstehen. Dann wirkt weiter der Arsenwasserstoff auf das DreifachChlorquerksilberarsin derartig ein, dass unter Austritt von Salzsaure Arsenquecksilber $\mathrm{As}^{2} \mathrm{Hg}^{3}$ gebildet wird. Einfach-Chlorquecksilberarsin, $\mathrm{As} \mathrm{H}^{2} \mathrm{Hg} \mathrm{Cl}$, entsteht nach der Gleichung

$$
\mathrm{AsH}^{3}+\mathrm{Hg} \mathrm{Cl}^{2}=\mathrm{HCl}+\mathrm{AsH}^{2} \mathrm{HgCl}
$$

als blassgelber Niederschlag, wenn viel Quecksilberchlorid im Ueberschuss vorhanden ist.

Durch längere Berührung mit einer Lösung von überschüssigem Quecksilberchlorid entstehen arsenige Säure, Salzsäure und Quecksilberchlorür.

$$
\left.4 \mathrm{AsH}^{2} \mathrm{HgCl}+14 \mathrm{Hg} \mathrm{Cl}^{2}+12 \mathrm{H}^{2} \mathrm{O}=4 \mathrm{AsH}^{8} \mathrm{O}^{8}+20 \mathrm{HCl}+9 \mathrm{Hg}^{2} \mathrm{Cl}^{2} .{ }^{1}\right)
$$

Leitet man länger Arsenwasserstoff ein, so bildet sich ein reich-

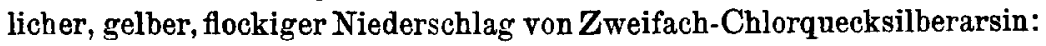

$$
\left.\mathrm{As}^{2} \mathrm{HgCl}+\mathrm{HgCl}^{2}=\mathrm{HCl}+\mathrm{AsH}(\mathrm{Hg} \mathrm{Cl})^{2} .{ }^{2}\right)
$$

Unter dem Einfluss von überschüssiger Quecksilberchloridlösung bildet es ebenfalls arsenige Säure, Salzsäure und Quecksilberchlorür: $\mathrm{AsH}(\mathrm{Hg} \mathrm{Cl})^{2}+4 \mathrm{HgCl}^{2}+3 \mathrm{H}^{2} \mathrm{O}=\mathrm{As}^{3} \mathrm{H}^{3}+4 \mathrm{HCl}+3 \mathrm{Hg}^{2} \mathrm{Cl}^{2}$.

Als drittes Einwirkungsprodukt des Arsenwasserstoffs auf Quecksilberchlorid entsteht das braun gefärbte Dreifach-Chlorquecksilberarsin:

$$
\mathrm{AsH}(\mathrm{Hg} \mathrm{Cl})^{2}+\mathrm{Hg} \mathrm{Cl}^{2}=\mathrm{HCl}+\mathrm{As}\left(\mathrm{HgCl}^{8} .\right.
$$

Dieses wird durch Wasser leicht zersetzt, daher am besten aus alkoholischer Lösung gewonnen. Bei Gegenwart von Quecksilberchlorid verläuft die Zersetzung nach der Gleichung: $2 \mathrm{As}(\mathrm{HgCl})^{8}=3 \mathrm{Hg}^{2} \mathrm{Cl}^{2}$ $+2 \mathrm{As}^{8}{ }^{8}$ ) whhrend bei Abwesenheit des Quecksilberchlorids durch die Einwirkung des Wassers arsenige Săure, Salzsäure und Quecksilber

1) Vergl. Stromeyer, l. c.

2) Vergl. Franceschi, l. c.

B) Vergl. Lohmann, l. c. 
entstehen $\left.{ }^{1}\right): \quad \mathrm{As}(\mathrm{HgCl})^{3}+3 \mathrm{H}^{2} \mathrm{O}=\mathrm{AsO}^{3} \mathrm{H}^{3}+3 \mathrm{HCl}+3 \mathrm{Hg}$. Die Reaktion von Arsenwasserstoff auf Dreifach-Chlorquecksilberarsin endlich erfolgt nach der Gleichung:

$$
\mathrm{As}(\mathrm{Hg} \mathrm{Cl})^{3}+\mathrm{AsH}^{8}=3 \mathrm{HCl}+\mathbf{A s}^{2} \mathrm{Hg}^{8} \text {. }
$$

Nachdem so die Reihe der vom Arsenwasserstoff sich ableitenden Quecksilberabkömmlinge eine gewisse Vollständigkeit erreicht hat, dürfte es interessant sein, die entsprechenden Derivate des Ammoniaks, des Phosphorwasserstoffs und des Antimonwasserstoffs zum Vergleiche heranzuziehen. Es sind von den hierhergehörigen Verbindungen bisher bekannt:

\begin{tabular}{|c|c|c|c|}
\hline As H $H^{8}$ & $\mathrm{NH}^{\mathrm{a}}$ & $\mathrm{PH}^{3}$ & $\mathrm{SbH}^{\mathrm{B}}$ \\
\hline $\mathrm{As} \mathrm{H}^{2} \cdot \mathrm{Hg} \mathrm{Cl}$ & $\mathrm{NH}^{2} \cdot \mathrm{HgCl}$ & - & - \\
\hline $\mathrm{AsH}\left(\mathrm{Hg} \mathrm{Cl}^{2}\right.$ & - & - & - \\
\hline $\mathrm{As}(\mathrm{Hg} \mathrm{Cl})^{8}$ & - & $\mathrm{P}(\mathrm{Hg} \mathrm{Cl})^{8}$ & - \\
\hline $\mathrm{As}^{2} \mathrm{Hg}^{8}$ & $\mathrm{~N}^{2} \mathrm{Hg}^{3}$ & $\mathrm{P}^{2} \mathrm{Hg}^{3}$ & - \\
\hline
\end{tabular}

\section{Experimenteller Teil.}

\section{Einfach - Chlorquecksilberarsin. A.s $\mathrm{H}^{2} \mathrm{HgCl}$.}

$15 \mathrm{~g}$ Quecksilberchlorid wurden in ca. $300 \mathrm{ccm}$ Alkohol gelöst und in diese Lösung mit Wasserstoff stark verdünnter Arsenwasserstoff unter beständigem Umschütteln eingeleitet. Die klare Flüssigkeit färbte sich zunächst gelb und es schied sich allmählich ein flockiger, hellgelber Niederschlag ab. Das Einleiten wurde solange fortgesetzt, bis etwa $2 \mathrm{~g}$ Niederschlag entstanden waren.

Der Niederschlag war nicht rein gelblich, da sich in der Einleitungsröhre stets die dunkler gefärbten höheren Einwirkungsprodukte bilden, die von Zeit zu Zeit abfallen und den Niederschlag verunreinigen. Diese sind aber spezifisch schwerer, als das Einfach-Chlorquecksilberarsin. Durch Dekantieren war es daher möglich, den Körper von den Beimischungen zu trennen. Er wurde auf dem Saugfilter mit absolutem Alkohol ausgewaschen und im Vakuum getrocknet.

$1,096 \mathrm{~g}$ Substanz lieferten bei der Analyse 0,2625 $\mathrm{g}$ Arsen und 0,68 g Quecksilber.

$0,7625 \mathrm{~g}$ Substanz verbrauchten $26,6 \mathrm{ccm}$ 1/10-N.-Silberlösung zur Titration des vorhandenen Chlors.

1) Vergl. Rose, l. c. 
Gefunden :

$$
\begin{aligned}
& \mathrm{Hg}=62,04 \\
& \mathrm{As}=23,95 \\
& \mathrm{Cl}=12,38
\end{aligned}
$$

Berechnet

$\begin{array}{cc}\text { für As H} \mathrm{H}^{2}(\mathrm{Hg} \mathrm{Cl})^{2}: & \text { für As H( } \mathrm{Hg} \mathrm{Cl})^{2}: \\ 64,00 \% & 73,12 \% \\ 24,60 \% & 13,71 \% \\ 11,36 \% & 13,71 \%\end{array}$

Dass Stromeyer als erstes Einwirkungsprodukt beider Reagentien Quecksilberchlorür und arsenige Säure gefunden hatte, rührte daher, dass er jedenfalls den Niederschlag mit der überschüssigen Quecksilberchloridlösuncr stehen gelassen hatte, wobei sich die von ihm gefundenen Körper durch Umsetzung bildeten:

$4 \mathrm{AsH}^{2} \mathrm{HgCl}+14 \mathrm{Hg} \mathrm{Cl}^{2}+12 \mathrm{H}^{2} \mathrm{O}=4 \mathrm{AsH}^{3} \mathrm{O}^{8}+20 \mathrm{HCl}+9 \mathrm{Hg}^{2} \mathrm{Cl}^{2}$.

Der Versuch beweist die Richtigkeit von Stromeyer's Angabe. Lässt man das Einfach-Chlorquecksilberarsin mit überschüssiger Quecksilberchloridlösung stehen, so tritt in der That eine Umsetzung im Sinne der vorstehenden Gleichung ein. Man hat, wenn der Niederschlag weiss geworden ist, im Filtrat neben dem überschüssigen Quecksilberchlorid die Salzsäure und die arsenige Säure, während der ausgewaschene Rückstand auf dem Filter sich als reines Quecksilberchlorür erweist.

Leitet man weiter Arsenwasserstoff in eine alkoholische Lösung von Quecksilberchlorid ein, so bildet sich der reichliche, orangegelbe Niederschlag des Zweifach-Chlorque cksilberarsins, für welchen bereits Franceschi (l. c.) die Zusammensetzung As $\mathrm{H}(\mathrm{Hg} \mathrm{Cl})^{2}$ ermittelt hat. Es bildet sich nach der Gleichung

$$
\mathrm{As} \mathrm{H}^{2} \mathrm{HgCl}+\mathrm{HgCl}^{2}=\mathrm{HCl}+\mathrm{AsH}(\mathrm{HgCl})^{2} \text {. }
$$

Dieses Zweifach-Chlorquecksilberarsin wird durch überschüssige Sublimatlösung ebenso wie das Einfach - Chlorquecksilberarsin in arsenige Säure, Salzsäure und Quecksilberchlorür zerlegt.

$\mathrm{AsH}(\mathrm{Hg} \mathrm{Cl})^{2}+4 \mathrm{HgCl}^{2}+3 \mathrm{H}^{2} \mathrm{O}=\mathrm{As} \mathrm{H}^{8} \mathrm{O}^{3}+4 \mathrm{HCl}+3 \mathrm{Hg}^{2} \mathrm{Cl}^{2}$.

Bei fortgesetztem Winleiten von Arsenwasserstoff nimmt der Niederschlag einen braunen Farbenton an (Rose, l. c.). Einen bequemen Weg, zu erkennen, wann gerade die Bildung dieses braunen Körpers beendet ist, giebt Lohmann (1. c.) an. Er verteilt die alkoholische Quecksilberchloridlösung in zwei Flaschen und leitet das Gasgemisch durch beide Flaschen. Sobald in der zweiten Flasche eine Fällung $\mathrm{zu}$ entstehen beginnt, ist in der ersten die Bildung des Rose'schen Körpers beendet:

$$
\mathrm{AsH}(\mathrm{HgCl})^{2}+\mathrm{HgCl}^{2}=\mathrm{HCl}+\mathrm{As}(\mathrm{HgCl})^{3} .
$$


Die Zersetzung des Dreifach-Chlorquecksilberarsins durch Wasser und durch Sublimatlösung kann, wie schon oben erwähnt, durch die Gleichungen:

$$
\begin{aligned}
\mathrm{As}(\mathrm{HgCl})^{3}+3 \mathrm{H}^{2} \mathrm{O} & =\mathrm{As}^{3} \mathrm{H}^{3}+3 \mathrm{HCl}+3 \mathrm{Hg}(\mathrm{Rose}) \text { und } \\
2 \mathrm{As}(\mathrm{Hg} \mathrm{Cl})^{8} & =3 \mathrm{Hg}^{2} \mathrm{Cl}^{2}+2 \mathrm{As}(\text { Lohmann })
\end{aligned}
$$

ausgedrückt werden.

Unsere Beobachtungen stimmen hinsichtlich des Zweifach- und Dreifach-Chlorquecksilberarsin so vollständig mit den vorhandenen Litteraturangaben überein, dass wir die Angabe unserer analytischen Daten unterlassen können.

\section{Arsenquecksilber, Arsenamalgam.}

$$
\mathrm{As}^{2} \mathrm{Hg}^{8} \text {. }
$$

Die von Rose offenbar für irrtümlich gehaltene Beobachtung von Stromeyer (l. c.), dass das Endprodukt der Einwirkung des Arsenwasserstoffs auf Quecksilberchlorid ein Arsenamalgam sei, konnten wir vollauf vestätigen. Leitet man nämlich, wenn die Bildung des Dreifach-Chlorquecksilberarsins erfolgt ist, weiter einen flotten Strom von Arsenwasserstoff ein, so färbt sich der Niederschlag rein schwarz. Er setzt sich schliesslich in Folge seiner Schwere rasch zu Boden, während die Flüssigkeit volikommen klar erschien. Das Arsenamalgam bildet sich nach der Gleichung:

$$
\mathrm{As}(\mathrm{HgCl})^{3}+\mathrm{AsH}^{3}=3 \mathrm{HCl}+\mathrm{As}^{2} \mathrm{Hg}^{8} \text {. }
$$

Es folgt dies aus folgendem Versuch.

$2,71 \mathrm{~g}$ Quecksilberchlorid wurden in etwa $250 \mathrm{ccm}$ absolutem Alkohol gelöst und in die Lösung Arsenwasserstoff bis zur vollständigen Beendigung der Reaktion eingeleitet. Nach dem Auswaschen mit Alkohol und Trocknen im Vakuum stellte der Niederschlag ein schwarzes, amorphes Pulver dar, dessen Gewicht 2,5566 g betrug.

In der abfiltrierten Flüssigkeit konnte kein Quecksilber mehr nachgewiesen werden, dasselbe war mithin vollkommen in den Niederschlag hineingegangen. Die im Filtrat enthaltene Salzsäure verbrauchte zur Titration $19,9 \mathrm{ccm}$ Norm.-Kalilauge.

$$
\begin{array}{cc}
\text { Gefunden: } & \text { Berechnet } \mathrm{HCl} \text { aus } 2,71 \mathrm{~g} \mathrm{Hg} \mathrm{Cl}^{2}: \\
\mathrm{HCl}=0,72745 \mathrm{~g} . & 0,73 \mathrm{~g} .
\end{array}
$$

Der getrocknete Niederschlag wurde mit Salzsäure und Kaliumchlorat gelöst, von freiem Chlor befreit und die Lösung auf ein bestimmtes Volumen aufgefüllt. In einem aliquoten Teile der Flüssigkeit wurde das Arsen durch Destillation mit Eisenchlorür und Salzsäure nach Schneider und Beckurts bestimmt. Ein anderer Teil diente dazu, das Quecksilber mit phosphoriger Säure als Chlorür zu fällen, das nach dem Auswaschen jodometrisch bestimmt warde. Es wuiden so 0,5014 $\mathrm{g}$ As und $1,97 \mathrm{~g} \mathrm{Hg}$ gefunden. 


$\begin{array}{cr}\text { Gefunden: } & \text { Berechnet für } \\ \mathrm{As}=20,4 \% & 20,0 \% \\ \mathrm{Hg}=79,5 \% & 80,0 \%\end{array}$

Das Arsen ist in diesem Amalgam sehr leicht oxydierbar. Bei einem Versuche, den Körper im Trockenschrank nachzutrocknen, wurde eine bedeutende Gewichtszunahme konstatiert, die nur auf einer Oxydation zu arseniger Săure beruhen konnte. Zum Nachweis wurde der Niederschlag mit Wasser digeriert; im Filtrat war mittelst Schwefelwasserstoff in der That Arsen nachzuweisen.

Als Herr Gronover später (im Sommer) grössere Mengen des Arsenquecksilbers - meist aus $30 \mathrm{~g}$ Sublimat - darstellte, beobachteten wir mehrfach, dass der frisch bereitete Körper beim Herausnehmen aus dem Vakuumexsiccator sich spontan oxydierte. Dabei trat oft eine so beträchtliche Selbsterhitzung ein, dass aus der Porzellanschale, welche das Amalgam enthielt. Ströme von Dämpfen, aus arseniger Säure und Quecksilber bestehend entwichen; auch etwas Arsensäure konnte dabei nachgewiesen werden. Nach dem Erkalten war dann die ganze Schale mit Quecksilbertröpfchen und arseniger Säure beschlagen und der Rest des von Quecksilbertröpfchen durchsetzten Inhaltes hatte eine graue Farbe angenommen.

\section{Jeber hexaalkylierte Diarsoniumverbindungen.}

Von A. Partheil, E. Amort und A. Gronover.

Alkylverbindungen des Arsens bilden einen Bestandteil der sogenannten Cadet'schen arsenikalischen Flüssigkeit, welche Cadet im Jahre 1760 durch Destillation von Kaliumacetat und arseniger Säure erhielt, deren chemische Natur er aber nicht erkannte. Mit dem Studium dieser Flüssigkeit hat sich Bunsen ${ }^{1}$ ) eingehend beschäftigt und aus derselben das Alkarsin oder Kakodyloxyd isoliert.

$$
4 \mathrm{CH}_{8} \mathrm{COOK}+\mathrm{As}^{2} \mathrm{O}^{\mathrm{a}}=\mathrm{As}^{2}\left(\mathrm{CH}^{3}\right)^{4} \mathrm{O}+\mathrm{K}^{2} \mathrm{CO}^{3}+2 \mathrm{CO}^{2} \text {. }
$$

Aus letzterem stellte er das Kakodyl selbst dar, dem er die einfache Formel As $\left(\mathrm{CH}^{3}\right)_{2}$ zuschrieb. Er zeigte, dass dasselbe sich wie ein Radikal verhalte und führte es als eine weitere wichtige Stütze für die damals geltende Radikaltheorie auf.

1) Annalen 31, 175; 37, $1 ; 42,14 ; 46,1$. 
Bei dem Behandeln von metallischem Arsen mit Methyljodid erhielten Cahours und $\mathrm{Rich} \mathrm{e}^{1}$ ) eine Doppelverbindung von $\mathrm{As}\left(\mathrm{CH}^{\mathrm{B}}\right)^{4}$ $\mathrm{J}+\mathrm{AsJ}^{3}$, die beim Kochen mit Kalilange Tetramethylarsoniumhydroxyd ergab nach folgenden Gleichungen:

$$
2 \mathrm{As}+4 \mathrm{CH}^{8} \mathrm{~J}=\mathrm{As}\left(\mathrm{CH}^{3}\right)^{4} \mathrm{~J}+\mathrm{As}^{3} \text {. }
$$

$\mathrm{As}\left(\mathrm{CH}^{8}\right)^{4} \mathrm{~J}+\mathrm{As} \mathrm{J}^{8}+4 \mathrm{KOH}=4 \mathrm{KJ}^{3}+\mathrm{As} \mathrm{O}^{3} \mathrm{H}^{3}+\mathrm{As}\left(\mathrm{CH}^{3}\right)^{4} \cdot \mathrm{OH}$.

Ferner liessen Cahours und Riche ${ }^{2}$ ) Methyljodid auf Arsennatrium einwirken und erhielten neben wenig Trimethylarsin Tetramethylarsoniumjodid, aus dem bei der Destillation mit festem Kaliumhydroxyd Trimethylarsin erhalten wurde:

$$
\begin{aligned}
& \text { As } \mathrm{Na}^{8}+3 \mathrm{CH}^{8} \mathrm{~J}=\mathrm{As}\left(\mathrm{CH}^{8}\right)^{3}+3 \mathrm{NaJ} \\
& \mathrm{As} \mathrm{Na} \mathrm{a}^{8}+4 \mathrm{CH}^{8} \mathrm{~J}=\mathrm{As}\left(\mathrm{CH}^{8}\right)^{4} \mathrm{~J}+3 \mathrm{Na} \mathrm{J} \\
& \mathrm{As}\left(\mathrm{CH}^{8}\right)^{4} \mathrm{~J}+\mathrm{KOH}=\mathrm{As}\left(\mathrm{CH}^{3}\right)^{8}+\mathrm{CH}^{8} \mathrm{OH}+\mathrm{KJ} \text {. }
\end{aligned}
$$

Quecksilber verbindet sich mit Jodmethyl zu Quecksilbermethyljodid, $\mathrm{CH}^{3} \mathrm{HgJ}$, eine Reaktion, deren Kenntnis wir Frankland ${ }^{3}$ ) verdanken. Lässt man dagegen Alkyljodid aut Arsenamalgam $\mathrm{As}^{2} \mathrm{Hg}^{8}$, einwirken, so entsteht als Hauptprodukt das Quecksilberjodiddoppelsalz eines hexaalkylierten Diarsoniumjodids: As $\mathbf{R}^{3} \mathrm{~J}$ $A s R^{B} \mathrm{~J}$.

Man könnte diese Körper auffassen als die Additionsprodukte von zwei Molekülen Jodalkyl an die Kakodyle. Indessen hat Cahours ${ }^{4}$ ) gezeigt, dass bei der direkten Einwirkung von Jodmethyl auf Kakodyl nach der Gleichung:

$$
\mathrm{As}^{2}\left(\mathrm{CH}^{3}\right)^{4}+2 \mathrm{CH}^{3} \mathrm{~J}=\mathrm{As}\left(\mathrm{CH}^{3}\right)^{4} \mathrm{~J}+\mathrm{As}\left(\mathrm{CH}^{8}\right)^{2} \mathrm{~J}
$$

Tetramethylarsoniumjodid und Kakodyljodid gebildet werden.

In der Stickstoffreihe kennen wir den Hexaalkyldiarsoniumverbindungen analog zusammengesetzte Körper bisher noch nicht. Am nächsten dürfte denselben das Triathylazoniumjodid $\left(\mathrm{C}^{2} \mathrm{H}^{5}\right)^{\mathbf{a}} \mathrm{N}$ $\left(\mathrm{NH}^{3}\right) J$ von E. Fischer $\left.{ }^{5}\right)$ stehen.

Es ist bemerkenswert, dass nur die Jodide der Alkoholradikale, nicht aber die Bromide und Chloride, befähigt sind, mit Arsena malgam Diarsoniumverbindungen zu bilden. Die Reaktion ist ferner nicht anf die Monojodsubstitutionsprodukte der Methanreihe beschränkt. Auch diejenigen der Olefine reagieren in demselben Sinne. Von den Jodiden aromatischer Alkoholradikale liefern diejenigen ein Diarsoniumderivat, welche das Halogen in einer der Fettreihe angebörenden
1) Annalen 122, 192.
8) Annalen 92, 361 .
8) Annalen 85, 361 .
4) Annalen 122, 209.
5) Annalen 199, 316. 
Seitenkette enthalten, bei Monojodbenzol dagegen blieb die Reaktion aus. Wir haben die Reaktion bisher mit Methyl-, A ethyl-, Propyl-, Isopropyl-, Butyl-, Allyl- und Benzyljodid durchführen können. Die Mehrzahl dieser Alkyljodide wirkt erst bei höherer Temperatur im geschlossenen Rohre auf Arsenamalgam ein, nur mit Allyljodid lässt sich die Reaktion bei Wasserbadwärme im mit Rückflusskühler versehenen Kolben zu Ende führen. Wir haben daher die Einwirkung dieses Jodids auf das Arsenamalgam einem eingehenderen Studium unterworfen, um dabei den Verlauf der Reaktion in seinen Einzelheiten festzustellen. Es gelang, den experimentellen Nachweis zu führen, dass die Reaktion nach folgender Gleichung verläuft:

$\mathrm{As}^{2} \mathrm{Hg}^{8}+8 \mathrm{C}^{8} \mathrm{H}^{8} \mathrm{~J}=\left[\mathrm{As}^{2}\left(\mathrm{C}^{8} \mathrm{H}^{5}\right)^{6} \mathrm{~J}^{2}+2 \mathrm{Hg} \mathrm{J}^{2}\right]+\mathrm{Hg} \mathrm{J}^{2}+\left[\mathrm{C}_{3} \mathrm{H}_{5}\right]_{8}$.

Daneben verläuft in nicht erheblichem Umfange eine Spaltung von Allyljodid, welche zur Bildung geringer Mengen Jodwasserstoff und eines gasförmigen, ungesättigten Koblenwasserstoffs führt, der kaum etwas anderes als Allylen sein kann.

Behandelt man ein Hexaalkyldiarsoniumquecksilberjodid mit überschüssigem, frisch gefälltem Chlorsilber, so entsteht ein Quecksilberchloriddoppelsalz des Hexaalkyldiarsoniumjodids, indem nur das Jod der beiden Quecksilberjodidmoleküle gegen Chlor vertauscht wird.

$\left[\mathrm{As}^{2} \mathrm{R}^{6} \mathrm{~J}^{2}+2 \mathrm{Hg} \mathrm{J}^{2}\right]+4 \mathrm{AgCl}=4 \mathrm{AgJ}+\left[\mathrm{As}^{2} \mathrm{R}^{6} \mathrm{~J}^{2}+2 \mathrm{Hg} \mathrm{Cl}^{2}\right]$.

Durch Einwirkung von feuchtem Silberoxyd auf die Quecksilberjodiddoppelsalze werden dagegen sămtliche Jodatome als Jodsilber entfernt und es entstehen stark alkalisch reagierende Lösungen der hexaalkylierten Diarsoniumhydroxyde, welche leicht Kohlensäure aus der Lnft anziehen.

$\left[\mathrm{As}^{2} \mathrm{R}^{6} \mathrm{~J}^{2}+2 \mathrm{Hg} \mathrm{J}^{2}\right]+3 \mathrm{Ag}^{2} \mathrm{O}+\mathrm{H}^{2} \mathrm{O}=\mathrm{As}^{2} \mathrm{R}^{6}(\mathrm{OH})^{2}+6 \mathrm{Ag} \mathrm{J}+2 \mathrm{HgO}$.

Die Basen selbst konnten bisher nicht in analysierbarem $\mathbf{Z u}$ stande erhalten werden. Sie dienten dazu, einige Salze und Doppelsalze daraus darzustellen.

Bei dem Versuche, das Hexapropyldiarsoniumbydroxyd durch Destillation im Vakuum zu reinigen, machten wir die Beobachtung, dass die Base bei dieser Operation eine Zersetzung erleidet. Das weitere Studium dieses Vorgangs führte zu der Erkenntnis, dass diese Spaltung nach der Gleichung erfolgt:

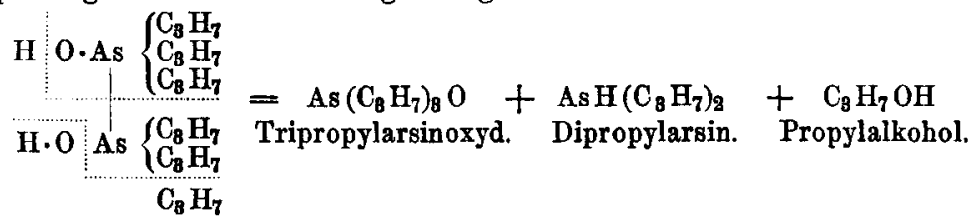

Arch. d. Pharm. CCXXXVII. Bds. 2, Heft. 
Das Tripropylarsinoxyd konnte in Form seiner gut krystallisierenden Quecksilberchloridverbindung isoliert werden. Das Dipropylarsin wurde durch seine reduzierende Wirkung und sein Oxydationsprodukt, die Dipropylarsinsäure oder Propylkakodylsäure, As $\left(\mathrm{C}_{8} \mathrm{H}_{7}\right)^{2} \cdot \mathrm{O} \cdot \mathrm{OH}$ charakterisiert.

Von den im nachstehenden beschriebenen Versuchen hat Herr Apotheker Dr. E. Amort die Jodderivate der Methanreihe, Herr Apotheker A. Gronover das übrige bearbeitet. An den Versuchen über die Spaltung des Hexapropyldiarsoniumbydroxyds sind beide Herren thätig gewesen.

\section{Experimenteller Teil.}

Das zu den folgenden Versuchen verwendete Quecksilberarsen $\mathrm{As}^{2} \mathrm{Hg}^{3}$ stellten wir, wie in der vorhergehenden Abhandlung auseinandergesetzt ist, her. Der Arsenwasserstoff wurde aus einem Kipp'schen Apparat entwickelt, der mit Zink und verdünnter Salzsäure beschickt war, in welcher ungefähr $1 \%$ arseniger Säure gelöst war. Der arsenwasserstoff haltige Wasserstoff wurde gewaschen und getrocknet und dann in zwei hintereinander geschaltete Flaschen geleitet, welche $30 \mathrm{~g}$ Quecksilberchlorid, gelöst in etwa $1 \frac{1}{2} l \mathrm{Alkohol}$, enthielten. Das Einleiten wurde unter häufigem Umschütteln fortgesetzt, bis der entstandene Niederschlag eine rein schwarze Farbe angenommen hatte und sich rasch zu Boden setzte, während die überstehende Flüssigkeit vollkommen klar erschien. lie Abgase wurden direkt ins Freie geleitet. Die Gewissheit, dass die Reaktion völlig zu Ende geführt war, wurde dadurch gewonnen, dass die gebildete Salzsäure im Filtrate mit Normal-Kalilauge titriert wurde. Das mit Alkohol ausgewaschene, im Vakuumexsiccator getrocknete Arsenamalgam muss in möglichst frisch bereitetem Zustande verwendet werden, wie oben ausgefïhrt wurde.

\section{Einwirkung von Aethylchlorid, Aethylbromid und Propylbromid auf Arsenamalgam.}

Die drei Halogenalkyle wurden mit etwas weniger als der berechneten Menge Arsenamalgam in Druckröhren eingeschmolzen und zunächst 5 Stunden im Wasserbade erhitzt. Da keine Einwirkung zu beobachten war, wurde das das Chloräthyl enthaltende Rohr weitere 
8 Stunden im Wasserbade, die anderen beiden Rohre im Schiessofen erst auf $120^{\circ}$, dann auf $180^{\circ}$ erhitzt. In allen Fällen wurde das gewünschte Resultat nicht erzielt, die Chloride und Bromide der Alkoholradikale vermögen mit Arsenamalgam nicht unter Bildung von Diarsoniumverbindungen zu reagieren.

\section{Einwirkung von Propyljodid auf Arsenamalgam.}

Arsenamalgam wurde mit dem doppelten Gewicht Propyljodid in ein Druckrohr eingeschmolzen und im Schiessofen 3 Stunden auf $180^{\circ}$ erhitzt. In dem erkalteten Rohre zeigten sich drei Schichten. Die oberste Schicht bestand aus überschïssigem Propyljodid und aus Kohlenwasserstoff, der beim Oeffnen des Rohres entwich und mit stark leuchtender, russender Flamme verbrannte.

Die mittlere, nur etwa $1 \mathrm{~mm}$ hohe Schicht, bestand aus einer stark rauchenden, sauren Flüssigkeit, welche die Reaktionen der Jodwasserstoffsäure zeigte. Näher charakterisiert wurde aber weder diese, noch die oben erwähnte Flüssigkeit.

Die untere Schicht, welche bei weitem die Hauptmenge des Reaktionsproduktes ausmachte, bildete eine braune, schmierige Masse, aus der sich beim Behandeln mit Aether ein gelbes Pulver in reichlicher Menge abschied. Der weitere Verlanf unserer Untersuchungen hat gezeigt, dass wir in demselben das

\section{Hexapropyldiarsoniumquecksilberjodid}

$$
\mathrm{As}^{2}\left(\mathrm{C}^{3} \mathrm{H}^{7}\right)^{6} \mathrm{~J}^{2}+2 \mathrm{Hg} \mathrm{J}^{2}
$$

vor uns hatten. Dieses Pulver worde mit Aether gewaschen, bis derselbe fast farblos abliet. Das so gereinigte Pulver löste sich schwer in kaltem, leicht in heissem Alkohol und krystallisierte daraus beim Erkalten in schönen, langen, gelben Nadeln oder in federbartartigen Büscheln.

Der Schmelzpunkt dieser Krystalle lag bei $120^{\circ}$. Die Analyse der Substanz machte anfärglich grosse Schwierigkeiten, welche bezüglich der Kohlenstoff bestimmung auch nicht behoben werden konnten. Herr Dr. P. Fritsch, Marburg, hatte die Liebenswürdigkeit, nach der von ihm abgeänderten Messinger'schen Methode der Kohlenstoffbestimmung auf nassem Wege drei Bestimmungen auszuführen, welche indessen ebenfalls untereinander bedeutend abweichende Werte lieferten. Die Bestimmung des Jods führte weder nach Carius, noch durch Glühen mit Natriumkarbonat zum Ziele; es wurden Werte erhalten, welche zwischen 46,4 und $47,8 \%$ schwankten. Dagegen wurden durch 
Digerieren der in Alkohol gelösten Substanz mit überschüssigem feuchten Silberoxyd brauchbare Werte erhalten. Zur Bestimmung von Arsen und Quecksilber wurde die Substanz nach Carius zerstört, das Arsen nach Schneider und Beckurts bestimmt, das Quecksilber als Schwefelquecksilher gefällt und als Chlorür gewogen.

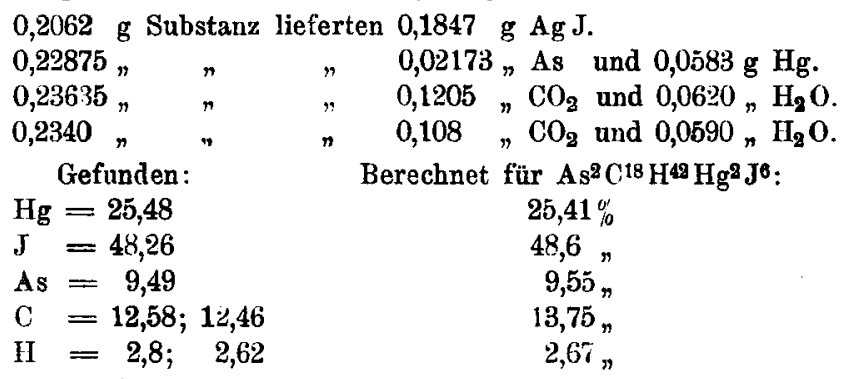

Dass trotz des zu niedrig gefundenen Kohlenstoffgehaltes dennoch die der Berechnung zu Grunde gelegte Formel richtig ist, geht aus der Analyse des später zu erwähnenden Quecksilberchloriddoppelsalzes hervor.

\section{Hexapropyldiarsoniumjodid - Quecksilberchlorid}

$$
\mathrm{As}^{2}\left(\mathrm{C}^{3} \mathrm{H}^{7}\right)^{6} \cdot \mathrm{J}^{2}+2 \mathrm{Hg} \mathrm{Cl}^{2} \text {. }
$$

Als uns die Carius'sche Methode sowie das Glühen mit Natriumkarbonat ungenügende Resultate bei der Jodbestimmung in dem Hexapropyldiarsoniumquecksilberjodid ergeben hatte, versuchten wir fiür diesen Zweck die Eigenschaft des frisch gefällten Chlorsilbers zu benutzen, sich mit den Jodverbindungen zu Jodsilber umzusetzen.

Das aus $15 \mathrm{ccm} 1 / 10$-Norm. - Silberlösung erhaltene Chlorsilber wurde mit Alkohol in die alkoholische Lösung von 0,2345 g Hexapropyldiarsoniumquecksilberjodid gebracht. Nach einstündigem Erhitzen auf dem Wasserbade wurde durch das vorher bereits zum Auswaschen des Chlorsilbers benutzte gewogene Filter filtriert und der Niederschlag bei $100^{\circ}$ getrocknet.

Es wurden $0,271 \mathrm{~g}$ Halogensilber gewogen, entsprechend $0,07738 \mathrm{~g}$ Jod $=32,99 \%$. Es waren mithin nur zwei Drittel des vorbandenen Jods durch das Chlorsilber eliminiert worden, die beiden am Arsen stehenden Jodatome aber intakt geblieben. Die Reaktion erfolgte also nach der Gleichung:

$\left[\mathrm{As}_{2}\left(\mathrm{C}^{9} \mathrm{H}^{7}\right)^{6} \mathrm{~J}^{2}+2 \mathrm{Hg} \mathrm{J}^{2}\right]+4 \mathrm{AgCl}=4 \mathrm{Ag} \mathrm{J}+\left[\mathrm{As}_{2}\left(\mathrm{C}^{8} \mathrm{H}^{7}\right)^{6} \mathrm{~J}^{2}+2 \mathrm{Hg} \mathrm{Cl}^{2}\right]$.

Das Filtrat lieferte nach dem Eindampfen das Quecksilberchloriddoppelsalz in schönen, nadelförmigen, weissen Krystallen, in denen sich das Jod nach dem Glühen mit Natriumkarbonat nachweisen liess. 


\section{Einwirkung von feuchtem Silberoxyd auf Hexapropyldiarsonium- quecksilberjodid. Hexapropyldiarsoniumhydroxyd.}

$$
\mathrm{As}_{2}\left(\mathrm{C}^{3} \mathrm{H}^{7}\right)^{6}(\mathrm{OH})^{2} \text {. }
$$

Feuchtes Silberoxyd wirkt auf Hexapropyldiarsoniumquecksilberjodid entsprechend folgender Gleichung ein:

$$
\begin{gathered}
{\left[\mathrm{As}^{2}\left(\mathrm{C}^{3} \mathrm{H}^{7}\right)^{6} \mathrm{~J}^{2}+2 \mathrm{Hg} \mathrm{J}^{2}\right]+\mathrm{As}^{2}\left(\mathrm{Cg}^{3} \mathrm{H}^{7}\right)^{6}(\mathrm{OH})^{2} .}
\end{gathered}
$$

Wir konnten daher die Reaktion zur Bestimmung des Jods in obigen Quecksilberjodiddoppelsalzen verwerten.

Die mit Silberoxyd versetzte, gelbgefärbte alkoholische Lösung des Quecksilberjodiddoppelsalzes trübt sich anfangs, wird aber nach etwa einstündigem Erwärmen auf dem Wasserbade farblos und klar. Die dann von dem Niederschlage abfiltrierte Lösung besitzt stark alkalische Reaktion. Die Alkalität entspricht der nach obiger Formel berechneten Stärke.

0,143 $\mathrm{g}$ Hexapropyldiarsoniumquecksilberjodid wurden in der soeben beschriebenen Weise mit Silberoxyd behandelt. Das Filtrat gebrauchte zur Titration $1,8 \mathrm{ccm} 1 / 10$-Norm.-Salzsäure, während sich 1,754 ccm berechnen. Die freie Diarsoniumbase zieht begierig Kohlensäure aus der Luft an; sie konnte bisher noch nicht in analysierbarer Form erhalten werden.

Ihr Chlorid, Oxalat und Jodid sind sebr leicht lösliche, farblose Salze. Das letztere schmilzt bei $150^{\circ}$ unter Braunfärbung.

\section{Hexapropyldiarsoniumquecksilberchlorid.$$
\mathrm{As}^{2}\left(\mathrm{C}^{8} \mathrm{H}^{7}\right)^{6} \mathrm{Cl}^{2}+2 \mathrm{Hg} \mathrm{Cl}{ }^{2} \text {. }
$$

In der wăsserigen Lösung des Hexapropyldiarsoniumchlorids entsteht auf Zusatz von kaltgesättigter Quecksilberchloridlösung ein weisser Niederschlag, der nach dem Absaugen und Auswaschen mit Wasser, aus Alkohol umkrystallisiert, schöne, weisse Krystallnadeln liefert. Das Salz schmilzt bei $169^{\circ}$.

Bei der Analyse lieferten: 0,2055 $\mathrm{g}$ Substanz $=0,029407 \mathrm{~g}$ As und $0,07998 \mathrm{~g} \mathrm{Hg}$.

$$
\begin{aligned}
& 0,274 \mathrm{~g} \text { Substanz }=0,057266 \mathrm{~g} \mathrm{Cl} \\
& 0,234, \quad=0,18206, \mathrm{CO}_{2} \text { und } 0,08665 \mathrm{~g} \mathrm{H}^{2} \mathrm{O} \text {. } \\
& \text { Gefunden: } \\
& \mathrm{Hg}=38,92 \% \\
& A s=14,31 \text {, } \\
& \mathrm{Cl}=20,90 \text { n } \\
& \mathrm{C}=21,22 \text {, } \\
& \text { Berechnet für } \mathrm{As}^{2} \mathrm{C}^{18} \mathrm{H}^{42} \mathrm{Hg}^{2} \mathrm{Cl} \text { : } \\
& \mathrm{H}=4,11 \text {, } \\
& 39,17 \% \\
& 14,68, \\
& 20,86 \text {, } \\
& 21,15 \text { n } \\
& 4,11 \text {, }
\end{aligned}
$$




\section{Hexapropyldiarsoniumplatinchlorid.}

$$
\mathrm{As}^{2}\left(\mathrm{C}^{3} \mathrm{H}^{7}\right)^{6} \mathrm{Cl}^{2}+\mathrm{PtCl}^{4} \text {. }
$$

Auf Zusatz einer konzentrierten Platinchloridlösung zu der wässerigen Lösung des Hexapropyldiarsoniumchlorids entsteht ein gelber Niederschlag, der nach dem Absaugen und Auswaschen aus heissem Wasser umkrystallisiert wurde. Aus konzentrierter Lösung krystallisiert das Doppelsalz in gelben Blättchen, beim langsamen Eindunsten einer kaltgesättigten wässerigen Lösung im Vakuum aber in gelben Säulen. Die Substanz schmilzt bei $189^{\circ}$.

Die Bestimmung des Platins durch einfaches Verglühen wurde dadurch unmöglich gemacht, dass die Substanz trotz grösster Vorsicht derartig überkletterte, dass die Tiegel äusserlich wie Platintiegel aussahen. Es musste daher die Bestimmung des Platins gleichzeitig mit der des Chlors durch Gluhen mit Natriumkarbonat im schwer schmelzbaren Rohre ausgeführt werden.

0,193 g Substanz lieferten 0,04j8 g Pt und 0,0501437 g Cl.

Gefunden:

$$
\begin{aligned}
& \mathrm{Cl}=25,98 \% \\
& \mathrm{Pt}=23,71,
\end{aligned}
$$

Berechnet für $\mathrm{As}^{2} \mathrm{C}^{18} \mathrm{H}^{22} \mathrm{PtCl}^{6}$ :

$26,1 \%$

$23,8 \check{a}$,

\section{Zersetzung des Hexapropyldiarsoniumhydroxyds durch trockene Destillation im Vakuum.}

Da bei der Darstellung grösserer Quantitäten der Base es nicht $\mathrm{zu}$ vermeiden war, dass sich kleine Mengen von Silberoxyd darin auflösten, gelang es nicht, durch Abdunsten der alkoholischen Lösung derselben die Base in reinem Zustande zu erhalten. Wir versuchten daher, das Hexapropyldiarsoniumhydroxyd durch Destillation im Vakuum zu reinigen. Wenn diese Operation auch nicht $\mathrm{zu}$ dem in Aussicht genommenen Ziele führte, so hat sie doch eine Reihe höchst beachtenswerter Resultate geliefert.

Bei einem Vakuum von $12 \mathrm{~mm}$ und einer Temperatur von $105^{\circ}$ fand eine Zersetzung statt und es destillierte bei $97^{\circ}$ eine klare, farblose Flüssigkeit über. Das ölig fliessende Destillat besass einen unangenehmen, äusserst widerlichen, starken, an alten Thran erinnernden Geruch. Beim Stehen an der Luft erstarrte es allmählich fast vollständig zu einer farblosen, krystallinischen, sauer reagierenden Masse. Der krystallinische Rückstand löste sich leicht in Wasser, Alkohol und Aether, sehr schwer in kaltem, leichter in heissem Ligroin. Aus letzterem mehrmals umkrystallisiert, stellte der Körper voluminöse, 
farblose, glänzende, vollkommen geruchlose Blättchen oder Nädelchen dar, deren Schmelzpunkt bei $123^{\circ}$ liegt. Die wässerige Lösung reagiert stark sauer. Die Ausbeute ist eine geringe.

Die Verbrennung liess sich ohne Schwierigkeit ausführen.

$0,1666 \mathrm{~g}$ Substanz lieferten $0,2258 \mathrm{~g} \mathrm{CO}_{2}$ und $0,1035 \mathrm{~g} \mathrm{H}_{2} \mathrm{O}$.

$0,202 " n \quad 0,276, \mathrm{CO}_{2}$ und $0,1265, \mathrm{H}_{2} \mathrm{O}$.

Nach der Carius'schen Methode liess sich der Körper nicht glatt zerstören. Wir mussten daher behufs Bestimmung des Arsens die von Russe $1^{1}$ ) angegebene Methode anwenden, was in tolgender Modifikation geschah. Ein $40 \mathrm{~cm}$ langes, einerseits zugeschmolzenes Verbrennungsrohr wurde derart beschickt, dass dasselbe zunächst eine $1 \mathrm{~cm}$ lange Schicht reines Quecksilberoxyd, darauf eine Mischung aus gleichen Teilen Quecksilberoxyd und Natriumkarbonat mit der zu untersuchenden Substanz enthielt und hierauf das Rohr mit Natriumkarbonat, gemischt mit dem fünften Teil seines Gewichts Quecksilberoxyd, gefüllt wurde. Von dem offenen Ende des Rohres an wurde die Natriumkarbonatschicht in Zwischenräumen von $5 \mathrm{za} 5 \mathrm{~cm}$ erhitzt, sodass zwischen den erhitzten Stellen noch Quecksilberoxyd vorhanden war. Dann wurde die mit der Substanzmischung gefüllte Stelle erhitzt und schliesslich das ganze Rohr und das reine Quecksilberoxyd, bis die ganze Masse weiss war. Die entweichenden Quecksilberdämpfe wurden, um nicht davon belästigt zu werden, unter Wasser geleitet.

Der erkaltete Röhreninhalt wurde in Wasser gelöst, mit Salzsăure neutralisiert und auf ein bestimmtes Volum aufgefullt. In einem aljquoten Teil dieser Lösung wurde dann das Arsen nach Schneider und Beckurts bestimmt.

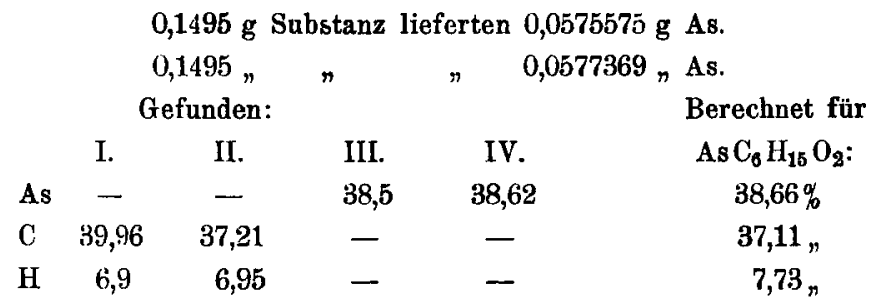

Der vorliegende Körper ist demnach zweifellos als Propyl. kakodylsäure $\mathrm{As}\left(\mathrm{C}_{3} \mathrm{H}_{7}\right)_{2} \mathrm{O} \cdot \mathrm{OH}$, anzusprechen.

Dis Propylkakodylsäure ist kein primäres Spaltungsprodukt bei der trockenen Destillation des Hexapropyldiarsoniumhydroxyds, sondern sie entsteht erst aus einem solchen durch Oxydation vermittelst des Luftsauerstoffes.

1) Annalen 107, 280. 
Wir haben daher, um zu verhindern, dass die Spaltungsprodukte bereits während der Destillation eine Oxydation erlitten, den Versuch im Wasserstoffstrom wiederholt. Als Zersetzungsgefäss diente ein Fraktionskolben, der in einem Oelbade auf $240^{\circ}$ erhitzt wurde.

Zuerst wurde der Alkohol, in dem das Hexapropyldiarsoniumhydroxyd gelöst war, bei möglichst niedriger Temperatur entfernt und alsdann die Vorlage durch kaltes Wasser gekühlt. Wiederum resultierte ein farbloses, öliges Destillat, das die cben beschriebenen äusseren Eigenschaften aufwies.

Ein Teil desselben wurde mit Silbernitratlösung versetzt. Es schied sich sogleich metallisches Silber aus, es musste mithin ein stark reduzierend wirkender Körper vorhanden sein.

Die Hauptmenge des Destillates wurde sofort in Alkohol gelöst und mit einer kalt gesättigten Quecksilberchloridlösung versetzt, bis sich dadurch keine weitere Ausscheidung bemerkbar machte.

Die ausgeschiedene amorphe Masse wurde auf der Nutsche gesammelt und mit Wasser und Alkohol ausgewaschen. Bei dem darauf folgenden Lösen in heissem Alkohol floss ein Teil des Körpers auf dem Boden des Gefässes zunächst zu einem Oel zusammen, löste sich aber auf Zusatz von mehr heissem Alkohol auf. Beim Stehen schieden sich aus dieser Lösung weisse Krystallnadeln aus, welche, nochmals aus Alkohol umkrystallisiert, bei $60-60,5^{\circ}$ schmolzen.

Zur Bestimmung des Quecksilbers wurde die alkoholisch-salzsaure Lösung der Substanz imit Schwefelwasserstoff behandelt und das Schwefelquecksilber nach Denner ${ }^{1}$ ) titriert.?

Berechnet für

1. 0,2081 g Substanz $=0,1085$ g $\mathrm{Hg}=52,14 \% \mathrm{Hg} \quad$ As $\left(\mathrm{C}_{8} \mathrm{H}_{7}\right)^{3} \mathrm{O}+2 \mathrm{Hg} \mathrm{Cl}^{2}$ :

2. $0,1846 \eta \quad=0,0962, \mathrm{Hg}=552,12, \mathrm{Hg} \quad 52,49 \% \mathrm{Hg}$

3. $0,2378 n,=0,0439, \mathrm{Cl}=18,47, \mathrm{Cl} . \quad 18,63, \mathrm{Cl}$

4. $0,2709 \eta n=0,0689, \mathrm{H}^{20}=2,82, \mathrm{H} \quad 2,76{ }_{n} \mathrm{H}$ und 0,14195 " $\mathrm{CO}^{2} \cong 14,28$, $\mathrm{C} \quad 14,17$, C.

Diese analytischen Werte beweisen, dass diese aus dem Destillat des Hexapropyldiarsoniumhydroxyds dargestellten Krystallnadeln das Quecksilberchloriddoppelsalz des Tripropylarsinoxyds waren. Die Propylbase erleidet also bei der Destillation eine Zersetzung, als deren eines Spaltungsprodukt Tripropylarsinoxyd nachgewiesen ist. Daneben entsteht ein stark reduzierend wirkender Körper. Nun wissen wir aus den Versuchen von Palmer ${ }^{2}$ ), dass das Dimethylarsin an

1) Pharm. Zentralh. 1888, 207.

2) Ber. 27, 1378. 
der Luft sogar unter Selbstentzündung sich oxydiert, dass es aus Silbernitratlösung metallisches Silber abscheidet und dass die Lösung dann jedenfalls Kakodylsäure enthält. In ganz analoger Weise vermag sich auch Dimethylphosphin zu Dimethylphosphinsüure zu oxydieren.

Man greift daher wohl nicht fehl, wenn man annimmt, dass der bei der Spaltung des Hexapropyldiarsoniumhydroxyds neben dem Tripropylarsinoxyd entstehende reduzierende Körper Dipropylarsin ist, welches dann bei der Oxydation die Dipropylarsinsäure (Propylkakodylsäure) liefert:

$$
\mathrm{AsH}\left(\mathrm{C}^{3} \mathrm{H}^{7}\right)^{2}+20=\mathrm{As}\left(\mathrm{C}^{3} \mathrm{H}^{7}\right)^{2} \cdot 0 \cdot 0 \mathrm{H} \text {. }
$$

Die Bildung von gasförmigen Zersetzungsprodukten, etwa von Kohlenwasserstoffen, konnte bei der Destillation der Propylbase nicht beobachtet werden. Es ist daher mit grosser Wahrscheinlichkeit anzunehmen, dass die Zersetzung des Hexapropyldiarsoniumhydroxyds bei der Destillation durch folgende Gleichung formuliert werden kann:

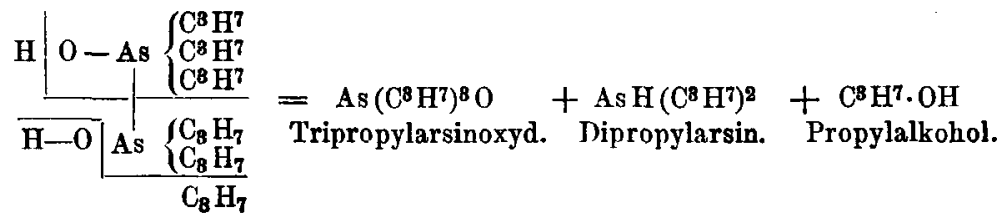

Diese Spaltungsgleichung erklärt in einfacher und bündiger Weise alle bisher bei der Destillation des Hexapropyldiarsoniumhydroxyds gemachten Beobachtungen. Es würde nur noch übrig bleiben, den Nachweis zu führen, dass in der That neben dem Tripropylarsinoxyd und dem Dipropylarsin noch Propylalkohol entsteht. Seiner geringen Menge halber, in der er zu erwarten ist, haben wir ihn bisher noch nicht fassen können.

\section{Diarsoniumderivate des Methyljodids. Hexamethyldiarsonium- quecksilberjodid. $\mathrm{As}^{2}\left(\mathrm{CH}^{8}\right)^{6} \mathrm{~J}^{2}+2 \mathrm{Hg} \mathrm{J}^{2}$.}

Arsenquecksilber wurde mit der doppelten Menge Jodmethyl in ein Druckrohr eingeschmolzen und im Schiessofen drei Stunden auf $120^{\circ}$ erhitzt. Das Reaktionsprodukt, bestehend aus einer krystallinischen Masse und einer darüber stehenden braunen Flüssigkeit, wurde getrennt, die Krystallmasse mit Aether extrahiert und aus siedendem Alkohol umkrystallisiert. Hierbei wird offenbar ein Teil der Substanz zersetzt, da dabei die Entwickelung eines unangenehm knoblauchartig riechenden, 
stark giftig wirkenden Gases sich bemerkbar macht. Aus der alkoholischen Lösung scheidet sich das Salz in unregelmässigen, gelben Krystallblättchen aus, welche nicht in Aether, schwer in kaltem, leichter in heissem Alkohol löslich sind und bei $184^{\circ}$ schmelzen.

$0,2150 \mathrm{~g}$ Substanz lieferten bei der Analyse 0,060866 g Hg.

Gefunden:

$\mathrm{Hg}=28,31 \%$
Berechnet für $\mathrm{As}^{2} \mathrm{C}^{6} \mathrm{H}^{18} \mathrm{Hg}^{2} \mathrm{~J}^{6}$ :

$28,53 \%$.

Die alkoholische Lösung des Salzes liefert beim Behandeln mit feuchtem Silberoxyd eine Lösung der freien Base $\mathrm{As}^{2}\left(\mathrm{CH}^{3}\right)^{6}(\mathrm{OH})^{2}$, welche mit Salzsäure das $\mathrm{Chlorid} \mathrm{As}^{2}\left(\mathrm{CH}^{3}\right)^{6} \mathrm{Cl}^{2}$ als strablig krystallinische, hygroskopische Masse liefert. Das Jodid $\mathrm{As}^{2}\left(\mathrm{CH}^{8}\right)^{6} \mathrm{~J}^{2}$ bildet weisse Krystallnadeln, die sich leicht in Wasser, schwerer in Alkohol lösen und bei $171^{\circ}$ unter Zersetzung schmelzen.

\section{Hexamethyldiarsoniumquecksilberchlorid.}

$$
\mathrm{As}^{2}\left(\mathrm{CH}^{8}\right)^{6} \mathrm{Cl}^{2}+2 \mathrm{Hg} \mathrm{Cl}^{2} \text {. }
$$

Versetzt man die wässerige Lösung des Hexamethyldiarsoniumchlorids mit kalt gesättigter Quecksilberchloridlösung, so entsteht ein weisser Niederschlag. Er bildet, nach dem Absaugen und Auswaschen mit Wasser aus Alkohol umkrystallisiert, schöne weisse Kıystallnadeln, welche den Schmelzpunkt $211^{\circ}$ zeigen.

Bei der Analyse lieferten 0,2115 g Substanz 0,09843 g Hg.

Gefunden:

$\mathrm{Hg}=46,54 \%$
Berechnet für $\mathrm{As}^{2} \mathrm{C}^{8} \mathrm{H}^{18} \mathrm{Hg}^{2} \mathrm{Cl}^{8}$ :

$46,89 \%$.

\section{Hexamethyldiarsoniumplatinchlorid.}

$$
\mathrm{As}^{2}\left(\mathrm{CH}^{3}\right)^{6} \mathrm{Cl}^{2}+\mathrm{PtCl}^{4} \text {. }
$$

In der konzentrierten wässerigen Lösung des Chlorids entsteht auf Zusatz einer konzentrierten Platinchloridlösung ein gelber Niederschlag, der nach dem Absaugen und Auswaschen aus Alkohol je nach den äusseren Bedingungen in gelben Blättchen oder Nadeln krystallisierte. Das Salz ist in Wasser schwer, leichter in Alkohol lösiich. Es schwärzt sich bei $245^{\circ}$ und schmilzt noch nicht bei $260^{\circ}$.

In $0,1975 \mathrm{~g}$ Substanz wurden $0,06454 \mathrm{~g} \mathrm{Cl}$ und $0,0589 \mathrm{~g}$ Pt gefunden.

Gefunden:

$\mathrm{Cl}=32,68 \%$

$\mathrm{Pt}=29,83$,
Berechnet für $\mathrm{As}^{2} \mathrm{C}^{8} \mathrm{H}^{18} \mathrm{PtCl}$ :

$32,89 \%$

30,04 , 
Abkömmlinge des Aethyljodids. Hexaäthyldiarsoniumquecksilberjodid.

$$
\mathrm{A} s^{2}\left(\mathrm{C}^{2} \mathrm{H}^{5}\right)^{6} \mathrm{~J}^{2}+2 \mathrm{Bg} \mathrm{J^{2 }} \text {. }
$$

Die Darstellung geschah aus Arsenamalgam und Jodäthyl durch dreistündiges Erhitzen im Druckrohr auf $180^{\circ}$. Dabei wurden dieselben Mengenverhältnisse und dieselbe Methode der Reinigung des Rohproduktes eingehalten, wie bei dem Jodmethyl ausgefühirt ist. Aus heissem Alkohol krystallisiert das Salz in nicht ganz regelmässigen Nadeln. Beim freiwilligen Verdunsten der alkoholischen Lösung bildeten sich wohlausgebildete säulenförınige Krystalle rom Schmelzpunkt $112^{\circ}$.

0,2050 g Substanz enthielten 0,05485 g $\mathrm{Hg}$.

$$
\begin{array}{cc}
\text { Gefunden: } & \text { Berechnet für } \mathrm{As}^{2}\left(\mathrm{C}^{2} \mathrm{H}^{5}\right)^{6} \mathrm{Hg}^{2} \mathrm{~J}^{8}: \\
\mathrm{Hg}=26,76 \% & 26,91 \% \%
\end{array}
$$

Die durch feuchtes Silberoxyd aus dem Quecksilberjodiddoppelsalz gewonnene Lösung der Base $\mathrm{As}^{2}\left(\mathrm{C}^{2} \mathrm{H}^{5}\right)^{6}(\mathrm{OH})^{2}$ lieferte beim Neutralisieren mit Salzsäure bezüglich Jodwasserstoffsäure Salze, von denen das Chlorid seiner Hygroskopizität halber nicht rein erhalten werden konnte. Das Jodid bildet farblose, nadelförmige Krystalle, leicht löslich in Wasser und Alkohol. Es schmilzt bei $162^{\circ}$ unter Zersetzung.

\section{Hexaãthyldiarsoniumquecksilberchlorid.$$
\mathrm{As}^{2}\left(\mathrm{C}^{2} \mathrm{H}^{5}\right)^{6} \mathrm{Cl}^{2}+2 \mathrm{Hg} \mathrm{Cl}^{2} \text {. }
$$

Das Salz krystallisiert, wie das entsprechende Methylderivat dargestellt, aus Alkohol in langen, farblosen Krystallnadeln. In Wasser lösen sie sich schwerer als in Alkohol und schmelzen bei $162^{\circ}$.

Bei der Analyse ergaben 0,2011 g Substanz 0,08587 g Hg.

$$
\begin{array}{cc}
\text { Gefunden: } & \text { Berechnet für } \mathrm{As}^{2} \mathrm{Cl}^{12} \mathrm{H}^{20} \mathrm{Hg}^{2} \mathrm{Cl}^{6}: \\
\mathrm{Hg}=42,7 \% & 42,68 \% .
\end{array}
$$

\section{Hexaāthyldlarsoniumplatinchlorid.}

$$
\mathrm{As}_{2}\left(\mathrm{C}_{2} \mathrm{H}_{5}\right)_{6} \mathrm{Cl}^{2} \cdot \mathrm{PtCl}^{4} \text {. }
$$

Das in ublicher Weise gewonnene Salz krystallisiert aus Wasser oder aus Alkohol in gelben Krystallblättchen oder in kleinen Krystallnadeln. Schmelzpunkt $237^{\circ}$.

Aus $0,196 \mathrm{~g}$ Substanz [wurden bei der Analyse $0,05688 \mathrm{~g}$ Gl and $0,0517 \mathrm{~g}$ Pt erhalten.

$$
\begin{array}{cc}
\text { Gefunden: } & \text { Berechnet für } \mathrm{As}^{2}\left(\mathrm{C}^{2} \mathrm{H}^{5}\right)^{6} \mathrm{PtCl}^{6} \\
\mathrm{Cl}=29,02{ }_{\circ}^{\circ} & 29,1 \% \\
\mathrm{Pt}=26,39{ }_{n} & 26,46 \%
\end{array}
$$




\section{Abkömmlinge des Isopropyljodids.}

Die Abkömmlinge des Isopropyljodids wurden genau wie die Derivate des Propyljodids dargestellt.

\section{Hexaisopropyldiarsoniumquecksilberjodid.}

$$
\mathrm{As}^{2}\left(\mathrm{C}^{8} \mathrm{H}^{7}\right)^{6} \mathrm{~J}^{2}+2 \mathrm{Hg} \mathrm{J}^{2} \text {. }
$$

Dieses Salz bildet, aus Alkohol umkrystallisiert, kleine blassgelbe Nadeln, leicht löslich in Alkohol, nicht in Aether. Der Schmelzpunkt liegt bei $114^{\circ}$.

Bei der Analyse lieferten 0,2210 g Substanz 0,05626 g Hg.

$$
\text { Gefunden: }
$$

$\mathrm{Hg}=25,46 \%$
Berechnet für $\mathrm{As}^{2}\left(\mathrm{C}^{3} \mathrm{H}^{7}\right)^{6} \mathrm{Hg}^{2} \mathrm{~J}^{\mathrm{a}}$ :

$25,41 \%$

Das Hexaisopropyldiarsoniumchlorid bildet zerfliessliche Nadeln. Das Jodid krystallisiert aus Alkohol in farblosen, nadelförmigen Krystallen, welche in Wasser, Alkohol und Aether sehr leicht löslich sind und bei $150^{\circ}$ unter Bräunung schmelzen.

\section{Hexaisopropyldiarsoniumquecksiberchlorid.}

$$
\mathrm{As} \mathrm{s}^{2}\left(\mathrm{C}^{3} \mathrm{H}^{7}\right)^{6} \mathrm{Cl}^{2}+2 \mathrm{Hg} \mathrm{Cl}{ }^{2} \text {. }
$$

Das Quecksilberchloriddoppelsalz krystallisiert aus Alkohol in Nadeln, welche leicht in Alkohol, schwer in Wasser löslich sind. Sie schmelzen bei $171^{\circ}$.

$0,2094 \mathrm{~g}$ Substanz ergaben bei der Analyse 0,08145 g Hg.

Gefunden:

$\mathrm{Hg}=38,9^{\circ}$
Bereclinct tür $\mathrm{As}^{2} \mathrm{C}^{18} \mathrm{H}^{42} \mathrm{Hg}^{2} \mathrm{Cl}^{6}$ :

$39,1 \%$.

\section{Hexaisopropyldiarsoniumplatinchlorid.}

$$
\mathrm{As}^{2}\left(\mathrm{C}^{3} \mathrm{H}^{5}\right)^{6} \mathrm{Cl}^{2} \cdot \mathrm{PtCl}^{4} \text {. }
$$

Das Platinsalz krystallisiert aus Alkohol in gelben Krystallen, aus Wasser in Blättchen. Es ist schwer löslich in kaltem. leicht löslich in heissem Wasser und Alkohol und schmilzt bei $211^{\circ}$.

$0,1830 \mathrm{~g}$ Substanz lieferten 0,047635 g Cl und 0,04345 g Pt bei der Analyse.

Gefunden:

$\mathrm{Cl}=26,03 \%$

$\mathrm{Pt}=23,74$,
Berechnet für $\mathrm{As}^{2} \mathrm{C}^{18} \mathrm{H}^{42} \mathrm{PtCl}^{8}$ :

$26,1 \%$

23,85 "

\section{Abkömmlinge des Norm. Butyljodids.}

Die Abkömmlinge des Norm. Butyljodids wurden ebenso, wie die im Vorstehenden beschriebenen Körper dargestellt, nur wurde das Arsenquecksilber mit der dreifachen Menge des Jodids in Reaktion gebracht. 


\section{Hexabutyldiarsoniumquecksilberjodid.}

$$
\mathrm{As}^{2}\left(\mathrm{C}^{4} \mathrm{H}^{9}\right)^{6} \mathrm{~J}^{2}+2 \mathrm{Hg} \mathrm{J}^{2} \text {. }
$$

Aus Alkohol umkrystallisiert, bildet dieses Doppelsalz gelbe, nadelförmige Krystalle, welche bei $109^{\circ}$ schmelzen.

$0,2300 \mathrm{~g}$ Substanz lieferten bei der Analyse 0,05365 g Hg. Gefunden: $\mathrm{Hg}=24,06 \%$ Berechnet für $\mathrm{As}^{2} \mathrm{C}^{24} \mathrm{H}^{54} \mathrm{Hg}^{8} \mathrm{~J}^{6}$ : $24,18 \%$.

Die aus der Butylverbindung gewonnene Base liefert ein hygroskopisches Chlorid und ein in schönen, farblosen Nadeln krystallisierendes Jodid, welches in Wasser und Alkohol leicht löslich ist und bei $146^{\circ}$ unter Zersetzung schmilzt.

\section{Hexabutyldiarsoniumquecksilberchlorid. $\mathrm{As}^{2}\left(\mathrm{C}^{4} \mathrm{H}^{9}\right)^{6} \mathrm{Cl}^{2}+2 \mathrm{Hg} \mathrm{Cl}^{2}$.}

Beim Zusammengiessen der Lösung des Hexabutyldiarsonium. chlorids mit gesättigter Quecksilberchloridlösung scheidet sich die Doppelverbindung in Form öliger Tropfen ab, die weder aus Wasser noch aus Alkohol krystallisiert werden konnten. Die alkoholische Lösung hinterliess beim Eindunsten im Vakuum eine strahlig-krystallinische Masse, welche nicht zu einer Analyse einlud.

\section{Hexabutyldiarsoniumplatinchlorid.}

$$
\mathrm{As}^{2}\left(\mathrm{C}^{4} \mathrm{H}^{9}\right)^{6} \mathrm{Cl}^{2} \cdot \mathrm{PtCl}^{4} \text {. }
$$

Das Platinsalz bildet gelbe Krystalle, welche in Wasser fast unlöslich sind, sich aber in Alkohol leicht lösen. Ihr Schmelzpunkt liegt bei $147^{\circ}$.

Die Platin- und Chlorbestimmung ergab aus 0,1791 g Substanz 0,04245 g Cl and $0,0384 \mathrm{~g}$ Pt.

$\begin{array}{cc}\text { Gefunden: } & \text { Berechnet für } \mathrm{As}^{2}\left(\mathrm{C}^{4} \mathrm{H}^{9}\right)^{6} \mathrm{Pt} \mathrm{Cl}^{8}: \\ \mathrm{Cl}=23,7 \% & 23,67 \% \\ \mathrm{Pt}=21,44 & 21,63\end{array}$

\section{Einwirkung von Allyljodid auf Arsenamalgam.}

Hexaallyldiarsoniumquecksilberjodid.

$$
\mathrm{As}^{2}\left(\mathrm{C}^{3} \mathrm{H}^{5}\right)^{6} \mathrm{~J}^{2}+2 \mathrm{Hg} \mathrm{J} \text {. }
$$

Arsenquecksilber reagiert mit dem doppelten Gewicht Allyljodid bereits im mit Rückflusskühler versehenen Kulben. Bisweilen trat die Reaktion spontan ein und die Mischung erhitzte sich so stark, dass das Allyljodid lebhaft zu sieden begann, stets aber konnte die Ein- 
wirkung durch schwaches Erwärmen auf dem Wasserbade leicht eingeleitet werden. Nach dem Entfernen des Kolbens vom Wasserbade setzt sich das Sieden des Allyljodids weiter fort, bis bei fleissigem Umschütteln fast das ganze Arsenamalgam umgesetzt ist. Das heisse Rohprodukt bildet dann eine klare, dickliche, braune Flüssigkeit, in der nur einige Partikelchen des Arsenamalgams herumschwimmen, die der Einwirkung entgangen sind. Beim Erkalten erstarrt dann fast der ganze Kolbeninhalt zu einer bräunlichen Krystallmasse. Die Reinigung geschah wie bei den oben beschriebenen Körpern mittelst Aether und dann wurde aus heissem Alkohol umkrystallisiert. Das Salz schmilzt beim Erhitzen unter dem Alkohol, bevor es sich darin auflöst. Aus der heissen Lösung scheidet es sich beim Abkühlen in gelben, farnblattähnlich gruppierten Krystallblättchen aus, die nach mehrmaligem Umkrystallisieren beim freiwilligen Verdunsten der alkoholischen Lösung sich als gelbe, vierseitige Tafeln ausschieden. In Alkohol sind die Krystalle schwer löslich, bedeutend leichter in Aceton, von dem sie etwa das gleiche Gewicht erfordern. Der Schmelzpunkt liegt bei $62,5-63^{0}$.

Die Bestimmung des Arsens geschah nach der Methode von Russel durch Glühen mit Natriumkarbonat und Quecksilberoxyd. Die Jodbestimmung wurde durch Digestion mit feuchtem Silberoxyd ausgeführt. Zur Quecksilberbestimmung wurde die Substanz mit Schwefelammonium digeriert und das Schwefelquecksilber als Chlorür gewogen.

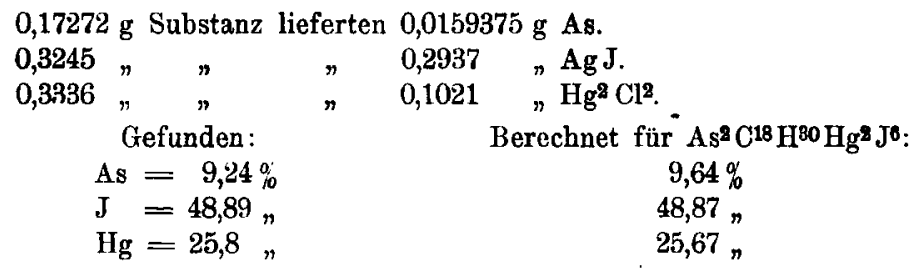

\section{Hexaallyldiarsoniumjodidquecksilberchlorid.}

$$
\mathrm{As}^{2}\left(\mathrm{C}^{8} \mathrm{H}^{5}\right)^{6} \mathrm{~J}^{2}+2 \mathrm{Hg} \mathrm{Cl}{ }^{2} \text {. }
$$

Digeriert maneinealkoholische Lösung des Hexaallyldiarsonium quecksilberjodids mit überschüssigem, frisch gefälltem Chlorsilber so erhält man ein farbloses Filtrat, aus welchem beim Verdunsten lange farblose Nadeln des Hexaallyldiarsoniumjodidquecksilber. chlorids krystallisieren. Der Schmelzpunkt des Salzes liegt bei $72,5^{\circ}$.

Die Reaktion verlăuft quantitativ. Das aus $15 \mathrm{ccm} 1 / 10$-Norm.Silberiösung dargestellte Chlorsilber lieferte, mit $0,3160 \mathrm{~g}$ des Quecksilberjodiddoppelsalzes digerier't, 0,2903 g Halogensilber. 


$$
\begin{array}{cc}
\text { Gefunden: } & \text { Berechnet: } \\
\mathrm{J}=32,92 \% & 32,58 \% .
\end{array}
$$

Das Hexaallyldiarsoniumhydroxyd, sein Chlorid sowie das Platin- und Quecksilberdoppelsalz konnten bisher nur als Oele gewonnen werden. Alle Versuche, diese Körper zu krystallisieren, schlugen fehl.

\section{Versuche, den Verlauf der Reaktion zwischen Arsenamalgam und Allyljodid zu ermitteln.}

Da die Reaktion zwischen Arsenamalgam und Allyljodid schon bei Wasserbadwärme glatt verläuft, ohne die Anwendung von Druckrohren zu erfordern, erschien uns dieselbe besonders geeignet, den Verlauf des Prozesses zu studieren. Die Entstehung des Hexaallyldiarsoniumquecksilberjodids kann man durch folgende Gleichung illustrieren:

$$
\mathrm{As}^{2} \mathrm{Hg}^{3}+6 \mathrm{C}^{3} \mathrm{H}^{5} \mathrm{~J}=\left[\mathrm{As}^{2}\left(\mathrm{C}^{8} \mathrm{H}^{5}\right)^{6} \mathrm{~J}^{2}+2 \mathrm{Hg} \mathrm{J}^{2}\right]+\mathrm{Hg} .
$$

Dieses Quecksilber wird aber nicht als solches abgeschieden, sondern es tritt weiter mit Allyljodid in Reaktion. Hierbei könnte entweder Quecksilberallyljodid entstehen:

$$
\mathrm{Hg}+\mathrm{C}^{8} \mathrm{H}^{5} \mathrm{~J}=\mathrm{C}^{8} \mathrm{H}^{5} \cdot \mathrm{Hg} \mathrm{J},
$$

oder es könnte sich Quecksilberjodid und Diallyl bilden:

$$
\mathrm{Hg}+2 \mathrm{C}^{3} \mathrm{H}^{5} \mathrm{~J}=\mathrm{Hg} \mathrm{J}^{2}+\left(\mathrm{C}^{3} \mathrm{H}^{5}\right)^{2} \text {. }
$$

Den Nachweis etwa entstandenen Quecksilberallyljodids versuchten wir zunächst darauf zu gründen, dass Hexaallyldiarsoniumquecksilberjodid in Aceton sehr leicht löslich ist, Quecksilberallyljodid beträchtlich weniger. Das vom überschüssigen Allyljodid befreite rohe Einwirkungsprodukt wurde daher in etwa gleichviel Aceton gelöst. Es blieben weissliche Flöckchen zurück, welche auf der Nutsche abgesogen und ausgewaschen wurden. Aus Alkohol krystallisierte der Körper in gelben, sechseckigen Täfelchen aus, die sich durch ihre äusseren Eigenschaften und ihren Schmelzpunkt als Jodoform charakterisierten.

Sodann versuchten wir etwa vorhandenes Allylquecksilberjodid in dem Rohprodukt dadurch nachzuweisen, dass wir es mit Cyankalium der Destillation im Wasserdampfstrom unterwarfen. Es hätte sich dann das dabei gebildete Diallyl im Destillat vorfinden müssen. Indessen schwamm auf diesem keine ölige Schicht und es zeigte auch nicht den charakteristischen Geruch des Diallyls. Es erscheint demnach ausgeschlossen, dass bei unserer Reaktion Quecksilberallyljodid gebildet wird. 
Um zu ermitteln, ob bei der Reaktion gasförmige Körper entstehen, wurde ein Versuch im Kohlensäurestrom ausgeführt und die den Apparat verlassenden Gase über Kalilauge aufgefangen. Es konnte so ein mit russender Flamme brennendes Gas aufgefangen werden, das von Bromwasser leicht absorbiert wurde, wobei sich einige Tröpfchen eines farblosen Oeles abschieden. Seine Menge war nicht so beträchtlich, dass man das Gas als ein wesentliches Produkt der Reaktion hätte ansprechen können. Es kann sich dabei um nichts anderes als um etwas Allylen, $\mathrm{C}_{8} \mathrm{H}_{4}$ gehandelt haben, das aus Allyljodid unter $\mathrm{Ab}$ spaltung von Jodwasserst off entstanden war.

Nunmehr wurde ein quantitativer Versuch in der Weise ausgeführt, dass der Apparat mit Wasserstoff gefüllt wurde; dann wurden zwei mit Fis gekühlte Vorlagen und schliesslich eine Vorrichtung angeschaltet. welche gestattete, das gebildete Allylen durch Messung des Volumens des verdrängten Wassers zu bestinmen und der Versuch in bekannter Weise durchgeführt. Das Rohprodukt wurde vom überschüssigen Allyljodid befreit, zunächst mit Wasser, dann mit einer Jodkaliumlösung von bekanntem Gehalt ausgezogen und in dem Wasser der Jodwasserstoff, in beiden Flüssigkeiten das gelöste Quecksilberjodid bestimmt. Dem Hexaallyldiarsoniumquecksilberjodid entzieht wässerige Jodkaliumlösung das Quecksilberjodid nicht. Angewendet wurden $18 \mathrm{~g}$ Quecksilberarsen und $36 \mathrm{~g}$ Allyljodid. Dabei wurden erhalten:

$180 \mathrm{ccm} \mathrm{C}^{8} \mathrm{H}^{4}$

$0,64 \mathrm{~g}$ H.J

1,2 $\mathrm{g} \mathrm{Hg} \mathrm{J}^{2}$, das durch den Jodwasserstoff gelöst wurde und

44,5 g mit Wasser ausgezogenes Rohprodukt. Letzteres wurde mit, Hilfe von Jodkaliumlösung zerlegt in

$10,75 \mathrm{~g} \mathrm{Hg} \mathrm{J}^{2}$ und

$33,75 \mathrm{~g}$ Quecksilberjodiddoppelsalz. Eine sehr geringe Menge Arsenamalgam war der Einwirkung entwangen.

Es waren also insgesamt $11,95 \mathrm{~g} \mathrm{Hg} \mathrm{J} \mathrm{J}^{2}$ entstanden. Verliefe die Einwirkung des Allyljodids auf Quecksilberarsen unter Bildung von Quecksilberallyljodid, so wäre kein Quecksilherjodid zu erwarten, während die Bildung von Diallyl das gleichzeitige Entstehen von $10,99 \mathrm{~g}$ Quecksilberjodid vorhersehen lässt. Das nicht als Quecksilberjodiddoppelsalz vorhandene Quecksilberjodid lässt sich dem Rohprodukt auch mittels Aether entziehen. Wir erhielten es daraus in schönen, roten Krystallen. Folgende Analyse dieses Quecksilberjodids beweist seine Reinheit. Es wurde in jodkaliumhaltigem Wasser gelöst und nach dem Ansäuern mit Salzsäure als Quecksilbersulfid gefällt.

$0,3025 \mathrm{~g}$ Substanz lieferten $0,1541 \mathrm{HgS}$. 


$$
\begin{gathered}
\text { Gefunden: } \\
\mathrm{Hg}=43,9 \%
\end{gathered}
$$

Berechnet für $\mathrm{Hg} \mathrm{J}^{2}$ : $44,05 \%$.

In den beiden mit Eis gekühlten Vorlagen fand sich eine geringe Menge einer Flüssigkeit vor, welche einen scharfen, dem Diallyl ähnlichen Geruch besass.

Durch diese Versuche ist bewiesen, dass die Einwirkung des Allyljodids auf Arsenamalgam unter Bildung von Quecksilberjodid und Diallyl verläuft. Letzteres kounte der geringen Menge wegen nicht in zur Analyse geeigneter Form erhalten werden. Trotzdem aber dürfte erwiesen sein, dass der Verlauf der Reaktion durch folgende Gleichung ausgedrückt werden muss:

$$
\mathrm{As}^{2} \mathrm{Hg}^{8}+8 \mathrm{C}^{3} \mathrm{H}^{5} \mathrm{~J}=\left[\mathrm{As}^{2}\left(\mathrm{C}^{8} \mathrm{H}^{5}\right)^{6} \mathrm{~J}^{2}+2 \mathrm{Hg} \mathrm{J}^{2}\right]+\mathrm{Hg} \mathrm{J}^{2}+\left(\mathrm{C}^{3} \mathrm{H}^{5}\right)^{2} \text {. }
$$

\section{Einwirkung von Quecksilberarsen auf Jodbenzoi.}

Quecksilberarsen wurde mit der dreifachen Menge Jodbenzol eingeschmolzen und zunächst mehrere Stunden auf $120^{\circ}$, darauf, weil keine Einwirkung erfolgt war, noch drei Stunden auf $150-180^{\circ}$ erhitzt. Beim Aufschmelzen entwichen grosse Mengen Jodwasserstoff. Aus dem schwärzlichen Inhalt des Rohres konnte viel Quecksilberjodid, aber keine Diarsoniumverbindung isoliert werden.

\section{Einwirkung von Quecksilberarsen auf Benzyljodid. Hexabenzyldiarsoniumquecksilberjodid.

$$
\mathrm{As}^{2}\left(\mathrm{C}^{6} \mathrm{H}^{5} \cdot \mathrm{CH}^{2}\right)^{6} \mathrm{~J}^{2}+2 \mathrm{Hg} \mathrm{J}^{2} \text {. }
$$

Im Druckrohr wurde das Arsenamalgam mit der dreifachen Menge Benzyljodid drei Stunden lang im Wasserbade erhitzt. Es hatten sich zwei Schichten gebildet. Die obere bestand aus einem braunen Oel, darunter befand sich noch ziemlich viel unverändertes Amalgam, durchsetzt mit gelbweissen Krystallblättchen. Beim Oeffnen des Rohres entwichen reichliche Mengen Jodwasserstoff. Nach dem Abtrennen des Oeles wurde der Rückstand mit Aether ausgezogen und aus heissem Alkohol krystallisiert. Beim Erkalten der alkoholischen Lösung schied sich zuerst eine weisslich-gelbe, harzartige Masse aus, erst später folgten schwach gelb gefärbte zarte Blättchen, bestehend aus Hexabenzyldiarsoniumquecksilberjodid. Die Ausbeute ist sehr bescheiden. Nach mehrmaligem Umkrystallisieren schmolz das Salz bei $163^{\circ}$.

Die mit Hilfe von feuchtem Silberoxyd ausgeführte Jodbestimmung ergab in 0,2531 g Sabstanz 0,1024 $\mathrm{g}$ Jod.

$$
\begin{array}{cc}
\text { Gefunden: } & \text { Berechnet für } \mathrm{As}^{2} \mathrm{C}^{\mathbf{4}} \mathrm{H}^{42} \mathrm{Hg}^{\mathbf{2}} \mathrm{J}^{6}: \\
\mathrm{J}=40,45 \% & 41,01 \% .
\end{array}
$$


Das feuchte Silberoxyd verwandelt die Lösung des Quecksilberjodiddoppelsalzes in die der freien Base. Beim Verdunsten, zuletzt im Vakuumexsiccator, hinterbleibt letztere als stark alkalischer Sirup, in welchem sich schliesslich Krystallnädelchen ausschieden. In analysenreiner Form konnte die Base aber bisher nirht erhalten werden. Sie wurde daher in das Chlorid verwandelt. Das Hexabenzyldiarsoniumchlorid vermag mit Krystallchloroform und mit Krystallwasser zu krystallisieren.

\section{Chlor oform-Hexabenzyldiarsoniumchlorid. $\mathrm{As}^{2}\left(\mathrm{C}^{6} \mathrm{H}^{5} \cdot \mathrm{CH}^{2}\right)^{6} \mathrm{Cl}^{2}+1 \frac{1}{2} \mathrm{CHCl}^{3}$.}

Setzt man die alkoholische Lösung des Quecksilberdoppelsalzes mit feuchtem Silberoxyd um, neutralisiert das Filtrat mit Salzsäure und verdunstet den Alkohol, so scheidet sich aus der zuletzt verbleibenden wässerigen Flüssigkeit das Chlorid als weissliche, harzartige Masse aus. Löst man dasselbe in Chloroform, versetzt die L Lösung mit Ligroin, bis eine schwache, milchige Trübung entsteht und nimmt man diese durch vorsichtigen Zusatz von Chloroform wieder weg, so krystallisiert beim Verdunsten des Lösungsmittels unter dem Vakuumexsiccator das Chlorid in sternförmig gruppierten, breiten, weissen, undurchsichtigen Nadeln aus, deren Schmelzpunkt zwischen $138^{\circ}$ bis $140^{\circ}$ liegt.

Die Krystalle geben intensive Isonitrilreaktion. Im Wassertrockenschrank bis zur Gewichtskonstanz getrocknet, verloren $0,5180 \mathrm{~g}$ Substanz 0,0935 g Chloroform.

Gefunden:

$\mathrm{CHCl}^{3}=18,1 \%$
Berechnet für $\mathrm{As}^{2} \mathrm{C}^{42} \mathrm{H}^{42} \mathrm{Cl}^{2}+1 \frac{1}{2} \mathrm{CHCl}$ : $18,9 \%$.

$0,3035 \mathrm{~g}$ der getrockneten Substanz lieferten, mit Natriumkarbonat geglüht, $0,027442 \mathrm{~g} \mathrm{Cl}$.

Gefunden:

$$
\mathrm{Cl}=9,04 \%
$$

Berechnet für $\mathrm{As}^{2} \mathrm{C}^{42} \mathrm{H}^{42} \mathrm{Cl}^{2}$ :

$$
9,25 \% \text {. }
$$

Das Chloroform ist an das Hexabenzyldiarsoniumchlorid nicht allzu fest gebunden. Bei längerer Auf bewahrung im Exsiccator dunstet ein Teil allmählich $a b$, sodass dann stimmende Analysenwerte nicht mehr gefunden werden. So enthielten beispielsweise 0,2430 längere Zeit im Chlorcalciumexsiccator aufbewahrter Substanz $0,052075 \mathrm{Cl}$ $=21,43 \% \mathrm{Cl}$, während für die Formel $\left[\mathrm{As}^{2} \mathrm{C}^{42} \mathrm{H}^{42} \mathrm{Cl}^{2}+1 \frac{1}{2} \mathrm{CH} \mathrm{Cl}{ }^{3}\right]$ $=24,12 \% \mathrm{Cl}$ sich berechnen. 


\section{Hexabenzyldiarsoniumchlorid. \\ $\mathrm{As}^{2}\left(\mathrm{C}^{6} \mathrm{H}^{5} \cdot \mathrm{CH}^{2}\right)^{6} \mathrm{Cl}^{2}+4 \mathrm{H}^{2} \mathrm{O}$.}

Aus der heiss bereiteten wässerigen Lösung des oben erwähnten Rohproduktes scheidet sich das Hexabenzyldiarsoniumchlorid mit vier Molekülen Krystallwasser aus. Nochmals aus heissem Wasser umkrystallisiert, bildet es leichte, weisse Nadeln, welche bei $142-142,5^{\circ}$ schmeizen. Sie besitzen einen aromatischen Geruch und sind mit den Wasserdämpfen etwas flüchtig.

Bei der Analyse lieferten 0,2743 g Substanz 0,0221875 Cl.

$0,3015 \mathrm{~g}$ verloren bei $105^{\circ}=0,02535 \mathrm{~g} \mathrm{H}^{2} \mathrm{O}$.

$0,2704 \mathrm{~g}$ getrockneter Substanz enthielten $0,02447 \mathrm{~g} \mathrm{Cl}$.

Gefunden:

Berechnet für

1. $8,09 \% \mathrm{Cl}$

2. $8,40{ }_{n} \mathrm{H}^{2} \mathrm{O}$

3. $9,05{ }_{n} \mathrm{Cl}$ $\mathrm{As}^{2} \mathrm{C}^{42} \mathrm{H}^{28} \mathrm{Cl}^{2}+4 \mathrm{H}^{2} \mathrm{O}: \quad \mathrm{As}^{2} \mathrm{C}^{49} \mathrm{H}^{42} \mathrm{Cl}^{2}$ :

\section{Hexabenzyldiarsoniumquecksilberchlorid.}

$\mathrm{As}^{2}\left(\mathrm{C}^{6} \mathrm{H}^{5} \cdot \mathrm{CH}^{2}\right)^{6} \mathrm{Cl}^{2}+2 \mathrm{Hg} \mathrm{Cl}^{2}$.

Auf Zusatz einer kalt gesättigten alkoholischen Quecksilberchloridlösung zu einer heissen konzentrierten Lösung des Hexabenzyldiarsoniumchlorids schieden sich nach dem Umschwenken plötzlich weisse Kryställchen aus, welche auf der Nutsche gesammelt, mit Alkohol gewaschen und aus heissem Alkohol umkrystallisiert wurden. Sie bildeten nun lange, weisse, schillernde Nadeln, die einen Schmelzpunkt von $175,5^{\circ}$ besassen.

Bei der Analyse lieferten $0,2894 \mathrm{~g}$ Substanz $0,04686 \mathrm{~g}$ Cl.

$$
\begin{aligned}
& 0,2421, \quad \text { \# } 0,03907 \text { n } \\
& 0,2851 \eta \quad 0,102, \mathrm{Hg}^{8} \mathrm{Cl}^{2} \text {. }
\end{aligned}
$$

Gefunden:

$$
\mathrm{Cl}=16,18 ; 16,13 \%
$$$$
\mathrm{Hg}=30,38 \%
$$

Berechnet fur $\mathrm{As}^{2} \mathrm{C}^{2} \mathrm{H}^{2} \mathrm{Hg}^{2} \mathrm{Cl}^{6}$ :

$16,27 \%$

30,55,

\section{Hexabenzyldiarsoniumplatinchlorid:}

$$
\mathrm{As}^{2}\left(\mathrm{C}^{6} \mathrm{H}^{5} \cdot \mathrm{CH}^{2}\right)^{6} \mathrm{Cl}^{2}+\mathrm{PtCl}^{4}+\mathrm{H}^{2} \mathrm{O} \text {. }
$$

Hexabenzyldiarsoniumchlorid in alkoholischer Lösung versetzten wir mit Platinchloridlösung im Ueberschuss. Es schieden sich farblose Nädelchen aus, die mit Alkohol gewaschen wurden und dann direkt zur Analyse verwendet wurden, weil sie nicht umkrystallisiert werden 
konnten. Beim Erbitzen mit Alkohol trat Reduktion ein. Das Salz schmolz unscharf bei $196-198^{\circ}$. Die Platin- und Chlorbestimmung wurde in der mit Natriumkarbonat erhitzten Substanz vorgenommen.

$0,2970 \mathrm{~g}$ Substanz lieferten $0,05552 \mathrm{~g} \mathrm{Cl}$.

$0,3044 \eta \quad$ verloren, bei $100^{\circ}$ getrocknet, $0,0056 \mathrm{~g} \mathrm{H}_{2} \mathrm{O}$.

Gefunden:

$$
\mathrm{Cl}=18,59 \%
$$$$
\mathrm{H}^{2} \mathrm{O}=1,80 \text {, }
$$

Berechnet für $\mathrm{As}^{2} \mathrm{C}^{42} \mathrm{H}^{42} \mathrm{PtCl}^{6}+\mathrm{H}^{2} \mathrm{O}$ :

$0,2988 \mathrm{~g}$ getrockneter Substanz enthielten $0,05198 \mathrm{~g}$ Pt.

Gefunden:

$\mathrm{Pt}=17,39 \underset{\%}{\alpha}$
Berechnet für $\mathrm{As}^{2} \mathrm{C}^{42} \mathrm{H}^{18} \mathrm{PtCl}^{8}$ :

$17,62 \%$.

\section{Hexabenzyldiarsoniumgoldchlorid.}

$$
\mathrm{As}^{2}\left(\mathrm{C}^{6} \mathrm{H}^{5} \cdot \mathrm{CH}^{2}\right)^{6} \mathrm{Cl}^{2}+2 \mathrm{AuCl}^{3} \text {. }
$$

Das in Chloroform gelöste Chloroform-Hexabenzyldiarsoniumchlorid wurde mit vielem kaltem Alkohol verdünnt und wässerige Goldchloridlösung im Ueberschuss zugefügt. Es schieden sich nach dem Umschütteln goldgelbe Krystallnädelchen aus, die nach dem Auswaschen aus heissem Alkohol umkrystallisiert wurden. Dabei trat aber Reduktion von metallischem Gold ein. Zur Analyse wurden deshalb die direkt gefällten Krystalle nach sorgfältigem Auswaschen verwendet. Beim Trocknen bei $100^{\circ}$ trat kein Gewichtsverlust ein. Nach dem Glühen mit Natriumkarbonat lieferten $0,1983 \mathrm{~g}$ Substanz $0,0417125 \mathrm{~g} \mathrm{Cl}$ und $0,0561 \mathrm{~g} \mathrm{Au}$.

$$
\begin{gathered}
\text { Gefunden: } \\
\mathrm{Cl}=21,03 \% \\
\mathrm{Au}=28,22 \%
\end{gathered}
$$

Berechnet für $\mathrm{As}^{2} \mathrm{C}^{42} \mathrm{H}^{42} \mathrm{Au}^{2} \mathrm{Cl}^{8}$ :

$20,69 \%$

28,61 ,

Bonn, den 9. Januar 1899. 